\title{
Proof of an Entropy Conjecture of Wehrl
}

\author{
Elliott H. Lieb*
}

Departments of Mathematics and Physics, Princeton University, Princeton, New Jersey 08540, USA

\begin{abstract}
Wehrl has proposed a new definition of classical entropy, $S$, in terms of coherent states and conjectured that $S \geqq 1$. A proof of this is given. We discuss the analogous problem for Bloch coherent spin states, but in this case the conjecture is still open. An inequality for the entropy of convolutions is also given.
\end{abstract}

\section{Introduction}

In a recent paper [1], A. Wehrl introduced a new definition of the "classical" entropy corresponding to a quantum system, proved that it had several interesting properties that deserve to be studied further, and posed a conjecture about the minimum value of this "classical" entropy. The main purpose of this paper is to prove Wehrl's conjecture. It is somewhat surprising that while the conjecture appears to be almost obvious, the proof we give requires some difficult theorems in Fourier analysis. The conjecture may or may not be important physically, but it reveals an interesting feature of coherent states.

To briefly recapitulate Wehrl's analysis, consider a single particle in one dimension, so that the Hilbert space is $L^{2}(\mathbb{R})$. (The generalization to $\mathbb{R}^{N}$ is trivial.) For each $z=(p, q) \in \mathbb{R}^{2}$, define the normalized vector $|z\rangle$ in $L^{2}(\mathbb{R})$ by

$$
|z\rangle \equiv(\pi \hbar)^{-1 / 4} \exp \left\{\left[-(x-q)^{2} / 2+i p x\right] / \hbar\right\} \equiv R(x \mid p, q) .
$$

These vectors are the coherent states used by Schrödinger [2], Bargmann [3], Klauder [4], and Glauber [5]. If

$$
P_{z}=|z\rangle\langle z|
$$

is the orthogonal projection onto $|z\rangle$ then

$$
\int \frac{d z}{\pi} P_{z}=I
$$

\footnotetext{
* Work partially supported by US National Science Foundation grant MCS 75-21684 A02
} 
where $d z / \pi \equiv d p d q / 2 \pi \hbar$ and $I=$ identity. The integral in (1.3) can be defined as a weak integral and (1.2) is simply the Plancherel equality.

For a "density matrix" $Q^{Q}($ a positive semidefinite operator of trace 1$)$ on $L^{2}(\mathbb{R})$, its quantum entropy is

$$
S^{Q}\left(Q^{Q}\right) \equiv-\operatorname{Tr} Q^{Q} \ln Q^{Q} \geqq 0 .
$$

The right side of (1.4) is well defined, although it may be $+\infty$.

For a nonnegative function $f$ on $\mathbb{R}^{2}$, with $\int f(z) d z / \pi=1$, its classical entropy is

$$
\tilde{S}(f)=-\int \frac{d z}{\pi} f(z) \ln f(z)
$$

In general this integral may not be well defined, but even if it is it can be negative.

Given a quantum density matrix $Q^{Q}$, Wehrl defines the function

$$
\varrho^{\mathrm{cl}}(z) \equiv\left\langle z\left|\varrho^{Q}\right| z\right\rangle,
$$

whence $0 \leqq Q^{\mathrm{cl}}(z) \leqq 1$. Then

$$
S^{\mathrm{cl}}\left(\varrho^{Q}\right) \equiv \tilde{S}\left(\varrho^{\mathrm{cl}}\right) \text {. }
$$

This is the classical entropy of $Q^{Q}$. [Note that by $(1.3), \int \varrho^{\mathrm{cl}}(z) d z / \pi=1$.] Since $0 \leqq Q^{\mathrm{cl}}(z) \leqq 1$, the integral in (1.5) is now well defined, and $S^{\mathrm{cl}} \geqq 0$.

The positivity of $S^{\mathrm{cl}}$ is one advantage of Wehrl's definition. On the contrary, if, as is usual, $Q^{Q}=Z_{Q}^{-1} \exp \left[-\beta\left(-\hbar^{2} \Delta / 2 m+V(q)\right)\right]$, the customary classical approximation is $f(z)=Z_{\mathrm{cl}}^{-1} \exp \left[-\beta\left(p^{2} / 2 m+V(q)\right)\right]$. The difficulty with $f$ is that $\tilde{S}(f)$ can be negative and, in general, $\tilde{S}(f) \rightarrow-\infty$ as $\beta \rightarrow \infty$.

A second advantage of Wehrl's definition is that $S^{\mathrm{cl}}$ is monotonic. If $Q_{12}^{Q}$ is a density matrix on $L^{2}(\mathbb{R}) \otimes L^{2}(\mathbb{R})$, and $\left|z_{1}, z_{2}\right\rangle \equiv\left|z_{1}\right\rangle \otimes\left|z_{2}\right\rangle$, one defines

$$
\varrho_{12}^{\mathrm{cl}}\left(z_{1}, z_{2}\right)=\left\langle z_{1}, z_{2}\left|\varrho_{12}^{Q}\right| z_{1}, z_{2}\right\rangle .
$$

One can then define $Q_{1}^{\mathrm{cl}}\left(z_{1}\right)$ by partial trace on 2 (either first on $Q_{12}^{Q}$ or else on the right side of (1.8); by (1.3) they are identical). Wehrl shows that the entropies satisfy

$$
S_{12}^{\mathrm{cl}} \equiv \tilde{S}\left(\varrho_{12}^{\mathrm{cl}}\right) \geqq \tilde{S}\left(\varrho_{1}^{\mathrm{cl}}\right) \equiv S_{1}^{\mathrm{cl}},
$$

in an obvious notation. This property, which is obviously desirable physically, does not hold in general for either the quantum entropies or for ordinary classical continuous entropies (see [6] for further details). It does hold for these particular classical entropies.

Not only is $S^{\mathrm{cl}} \geqq 0$, but Wehrl proves [1]

$$
S^{\mathrm{cl}}\left(Q^{Q}\right)>S^{Q}\left(Q^{Q}\right) \text {. }
$$

[To prove $\geqq$ note that $s(x)=-x \ln x$ is concave, so $s\left(\varrho^{\mathrm{cl}}(z)\right) \geqq\left\langle z\left|s\left(Q^{Q}\right)\right| z\right\rangle$. But $S^{Q}\left(\varrho^{Q}\right)=\int\left\langle z\left|S\left(\varrho^{Q}\right)\right| z\right\rangle d z / \pi$.] While the minimum of $S^{Q}$ is zero (for any pure state, i.e. one dimensional projection) the minimum of $S^{\mathrm{cl}}$ is not zero. Wehrl's conjecture is the following:

Theorem 1. The minimum of $S^{\mathrm{cl}}$ is 1 (independent of $\hbar$ ). This minimum occurs if $Q^{Q}=P_{z}$ for any $z$. 
Remarks. 1) There is no upper bound or lower bound (other than zero) for $S^{\mathrm{cl}}\left(\varrho^{Q}\right)$ $-S^{Q}\left(\varrho^{Q}\right)$.

2) It is easy to see from Theorem 1 that in $L^{2}\left(\mathbb{R}^{N}\right)$, the minimum of $S^{\mathrm{cl}}$ is $N$.

The proof of Theorem 1 will be given in Section II. An analogous conjecture can be posed for Bloch coherent spin states and this is discussed, but not proved, in Section III. In Section II an inequality (Theorem 3) on $L^{p}$ norms is also presented. Section IV contains an inequality which may be of use for related problems.

\section{Proof of Wehrl's Conjecture}

From now on we set $\hbar=1$. As a preliminary remark we note:

Lemma 2. If $Q^{Q}$ minimizes $S^{\mathrm{cl}}, Q^{Q}$ must be a pure state.

Proof. If $Q^{Q}=\sum_{i} \lambda_{i} \pi_{i}$, the $\pi_{i}$ being one dimensional orthogonal projections, $\lambda_{i}>0$ and $\sum \lambda_{i}=1$, then $\varrho^{\mathrm{cl}}(z)=\sum \lambda_{i} \varrho_{i}(z)$ with $\varrho_{i}(z)=\left\langle z\left|\pi_{i}\right| z\right\rangle$. By concavity of $\tilde{S}, \tilde{S}\left(\varrho^{\mathrm{cl}}(z)\right)$ $\geqq \sum \lambda_{i} \tilde{S}\left(\varrho_{i}\right)$, with equality if and only if $\varrho_{i}(z)=\varrho_{j}(z)$ almost everywhere for all $i, j$. Suppose $\varrho_{i}$ is a projection onto $\psi_{i} \in L^{2}(\mathbb{R})$. Let $w=q+i p \in \mathbb{C}$ and let $f_{i}(w)$ $=\int \psi_{i}(x) \exp \left[-x^{2} / 2+w x\right] d x$, which is an entire analytic function of $w[3]$. Then equality almost everywhere implies that $\left|f_{i}(w)\right|=\left|f_{j}(w)\right|$, all $w$, and hence $f_{i}(w)$ $=f_{j}(w) \exp (i \theta(w))$ and $\theta$ is real and analytic on the complement of the zeros of $f_{i}$. Hence, $\theta(w)=$ const. By the uniqueness of the Fourier transform, $\psi_{i}=\alpha \psi_{j}$, with $|\alpha|=1$, almost everywhere, and, hence $\pi_{i}=\pi_{i}$, which is a contradiction.

Thus, to prove Theorem 1 we have to consider

$$
f(p, q)=\int \psi(x) R(x \mid p, q) d x
$$

with $\|\psi\|_{2}=1$, and show that

$$
\tilde{S}\left(|f|^{2}\right) \geqq 1
$$

with equality if $\psi(x)=R(x \mid p, q)$ for some $(p, q)$.

We will first prove Theorem 3 which concerns $L^{p}$ norms of $f(p, q)$. Theorem 1 is a corollary of Theorem 3.

Theorem 3. Let $\psi \in L^{2}(\mathbb{R})$ with $\|\psi\|_{2}=1$, and $f$ given by (2.1) and (1.1). Then, for $s \geqq 2$

$$
I_{s} \equiv \iint \mid\left(\left.f(p, q)\right|^{s} d p d q / 2 \pi \leqq 2 / s\right.
$$

with equality for $s>2$ if $\psi(x)=\alpha R(x \mid p, q)$ for some $p, q$ and $|\alpha|=1$. For $s=2,(2.3)$ is an equality for all $\psi$.

To prove Theorem 3 we will require the following two lemmas (for $N=1$ ). The first (best constant in the Hausdorff-Young inequality) was proved by Beckner [7] and the second (best constant in Young's inequality) simultaneously by Beckner [7] and Brascamp and Lieb [8].

Lemma 4. Let $f \in L^{p}\left(\mathbb{R}^{N}\right), 1 \leqq p \leqq 2$, and $\hat{f}$ its Fourier transform $\left(\hat{f}(k)=\int f(x) e^{i k x} d x\right)$. Then, with $1 / p+1 / p^{\prime}=1$,

$$
\|\hat{f}\|_{p^{\prime}} \leqq\left\{C_{p}(2 \pi)^{1 / p^{\prime}}\right\}^{N}\|f\|_{p},
$$


where

$$
C_{p}^{2}=p^{1 / p}\left(p^{\prime}\right)^{-1 / p^{\prime}} \text { and } C_{1}=C_{\infty}=1 .
$$

Remark. Equality holds in (2.4) if $f$ is any Gaussian, i.e. $f(x)=a \exp \{-(x, M x)$ $+(x, b)\}, a \in \mathbb{C}, b \in \mathbb{C}^{N}$, and $M$ positive definite.

Lemma 5. Let $f \in L^{p}\left(\mathbb{R}^{N}\right), g \in L^{q}\left(\mathbb{R}^{N}\right) 1 \leqq p, q \leqq \infty$. Then, with $1+1 / r=1 / p+1 / q$, $r \geqq 1$, and $*=$ convolution,

$$
\|f * g\|_{r} \leqq\left\{C_{p} C_{q} / C_{r}\right\}^{N}\|f\|_{p}\|g\|_{q} .
$$

Equality holds [8] for $r>1$ and $N=1$ if and only if $f(x)=a \exp \left[-p^{\prime}(x-b)^{2}+i \delta x\right]$ and $g(x)=\alpha \exp \left[-q^{\prime}(x-\beta)^{2}+i \delta x\right]$ for some $a, \alpha \in \mathbb{C}$ and $\delta, b, \beta \in \mathbb{R}$. For $r=1$ (all $\left.N\right)$, $p=q=1$ and (2.5) is an equality for all positive $f, g$.

Remark. In the classical inequalities, $C_{p}$ is replaced everywhere by 1 in Lemmas 4 and 5.

Proof of Theorem 3. As a first step apply Lemma 4 (with $p^{\prime}=s$ ) to the function $g_{q}(x)=\psi(x) \pi^{-1 / 4} \exp \left[-(x-q)^{2} / 2\right]$, with $q$ regarded as a parameter. $\left(g_{q} \in L^{s^{\prime}}(\mathbb{R})\right.$ by Hölder's inequality.) Thus,

$$
\int|f(p, q)|^{s} d p / 2 \pi \leqq C_{s^{\prime}}^{s} \pi^{-s / 4} \phi_{s}(q)^{s / s^{\prime}},
$$

where $\phi_{\mathrm{s}}$ is the convolution

$$
\phi_{s}=|\psi(x)|^{s^{\prime}} * \exp \left[-s^{\prime} x^{2} / 2\right] \text {. }
$$

The second step is to integrate (2.6) over $q$ and use Lemma 5 with $p=q=2 / s^{\prime}$ and $r=s / s^{\prime}$. Since $\left\|\exp \left(-x^{2} / 2\right)\right\|_{2}=\pi^{1 / 4}, I_{s} \leqq\left\{C_{s^{\prime}}\left(C_{2 / s^{\prime}}\right)^{2 / s^{\prime}}\left(C_{s / s^{\prime}}\right)^{-1 / s^{\prime}}\right\}^{s}=2 / s$.

Equality holds in the first step if $\psi$ is any Gaussian. In the second step, since $p=q=2 / s^{\prime}$, equality holds for $s>2$ if $\psi$ is a Gaussian with the same variance as $\exp \left(-x^{2} / 2\right)$, which is the condition stated in the theorem. When $s=s^{\prime}=2$, equality for all $\psi$ is a simple consequence of the Plancherel formula.

Proof of Theorem 1. We continue to use the notation of Theorem 3. Let $\varepsilon>0$. Since $I_{2}=1, K_{\varepsilon}=\varepsilon^{-1}\left\{I_{2}-I_{2(1+\varepsilon)}\right\} \geqq(1+\varepsilon)^{-1}$ by Lemma 5. Assuming $\tilde{S}\left(|f|^{2}\right)<\infty$ (otherwise, there is nothing to prove), we claim that $\lim _{\varepsilon \downarrow 0} K_{\varepsilon}=\tilde{S}\left(|f|^{2}\right)$, which proves that $\tilde{S}\left(|f|^{2}\right) \geqq 1$. To see this note that by Theorem 3 or by the Schwarz inequality, $|f(p, q)| \leqq\|f\|_{\infty} \leqq 1$, and hence $0 \leqq \varepsilon^{-1}|f|^{2}\left\{1-|f|^{2 \varepsilon}\right\} \leqq-|f|^{2} \ln |f|^{2}$. Thus, $K_{\varepsilon} \rightarrow \tilde{S}\left(|f|^{2}\right)$ by dominated convergence.

\section{Bloch Coherent Spin States}

Instead of $L^{2}(\mathbb{R})$, one can consider the finite dimensional vector space $\mathscr{H}_{J}=\mathbb{C}^{2 J+1}$, $J=1 / 2,1,3 / 2, \ldots$. The analogue of the vectors $|z\rangle$ are the Bloch coherent states [913] in $\mathscr{H}_{J}^{\prime}$. These have been used to prove the classical limit of quantum spin systems [13]. For each unit vector $\Omega \in \mathbb{R}^{3}$, the vector $|\Omega\rangle \in \mathscr{H}_{J}$ is defined as the normalized vector (unique up to the phase) satisfying

$$
\Omega \cdot S|\Omega\rangle=J|\Omega\rangle,
$$


where $S=\left(S_{x}, S_{y}, S_{z}\right)$ are the usual angular momentum operators satisfying $\left[S_{x}, S_{y}\right]$ $=i S_{z}$ and cyclically. An explicit representation is

$$
\begin{aligned}
|\Omega\rangle & =\sum_{M=-J}^{J} A_{M}(\theta) \exp (-i M \phi)|M\rangle, \\
A_{M}(\theta) & =\left(\begin{array}{c}
2 J \\
M+J
\end{array}\right)^{1 / 2}[\cos (\theta / 2)]^{J+M}[\sin (\theta / 2)]^{J-M},
\end{aligned}
$$

where $(\theta, \phi)$ are the polar coordinates of $\Omega .|M\rangle$ is the normalized vector satisfying $S_{z}|M\rangle=M|M\rangle$ and whose phase is given by $|M\rangle=$ (pos. const.) $\left(S_{x}-i S_{y}\right)^{J-M}|J\rangle$. With the measure

$$
d \mu_{J}(\Omega)=(2 J+1) \sin \theta d \theta d \phi / 4 \pi
$$

on the unit sphere $S_{2}$, and

$$
P_{\Omega}=|\Omega\rangle\langle\Omega|
$$

the projection onto $|\Omega\rangle$, one has the analogue of (1.3):

$\int d \mu_{J}(\Omega) P_{\Omega}=I$.

Now given a density matrix $\varrho^{Q}$ on $\mathscr{H}_{J}$ one can imitate the Wehrl construction:

$$
Q^{\mathrm{cl}}(\Omega)=\left\langle\Omega\left|Q^{Q}\right| \Omega\right\rangle
$$

and $S^{\mathrm{cl}}\left(Q^{Q}\right)=\tilde{S}\left(Q^{\mathrm{cl}}\right)$ with

$$
\tilde{S}(f)=-\int f(\Omega) \ln f(\Omega) d \mu_{J}(\Omega) .
$$

The monotonicity of $S^{\mathrm{cl}}$ and the inequality $S^{\mathrm{cl}} \geqq S^{Q}$ carry over to this case.

It is easy to compute that since $[13]\left\langle\Omega^{\prime}\left|P_{\Omega}\right| \Omega^{\prime}\right\rangle=\left[\cos \frac{1}{2} \Theta\right]^{4 J}$, where $\Theta$ is the angle between $\Omega$ and $\Omega^{\prime}$,

$$
S^{\mathrm{cl}}\left(P_{\Omega}\right)=2 J /(2 J+1)
$$

The analogue of Theorem 1 is then

Conjecture. $S^{\mathrm{cl}}\left(Q^{Q}\right) \geqq 2 J /(2 J+1)$.

We will have to content ourselves with the following remarks.

Remark $A$. Suppose $\varrho^{Q}$ is of the form

$$
Q^{Q}=\int d \mu_{J}(\Omega) h(\Omega) P_{\Omega}
$$

with $h(\Omega) \geqq 0$ and $\int h d \mu_{J}=1$. Every $\varrho^{Q}$ can be written in the form $(3.10)$ with $h$ real but, for $J \geqq 1$, not necessarily with $h \geqq 0$, even though $Q^{Q}$ is positive. However $P_{\Omega}$ is of this form with $h$ being a delta function. By (3.10)

$$
\varrho^{\mathrm{cl}}(\Omega)=\int d \mu_{J}\left(\Omega^{\prime}\right)\left[\cos \frac{1}{2} \Theta\right]^{4 J} h\left(\Omega^{\prime}\right) .
$$

Since $\varrho^{\mathrm{cl}}(\Omega)$ is then a convex combination of $\left|\left\langle\Omega \mid \Omega^{\prime}\right\rangle\right|$ 's, the concavity of $\tilde{S}$ leads to

$$
S^{\mathrm{cl}}\left(Q^{Q}\right) \geqq S^{\mathrm{cl}}\left(P_{\Omega^{\prime}}\right)=2 J(2 J+1)
$$

if $h(\Omega) \geqq 0$. The analogue of this remark would, of course, also hold for the original Wehrl problem. 
Remark B. Lemma 2 holds for the Bloch case as well. Thus we can assume $Q^{Q}$ is a projection onto $\psi \in \mathscr{H}_{J}$. Then

$$
\begin{aligned}
\varrho^{\mathrm{cl}}(\Omega) & =|f(\Omega)|^{2} \\
f(\Omega) & =\sum_{M=-J}^{J} C_{M} A_{M}(\theta) e^{-i M \phi}
\end{aligned}
$$

and $\sum\left|C_{M}\right|^{2}=1$

If $J=1 / 2$, every $\psi=\alpha|\Omega\rangle$ for some $|\Omega\rangle$ and $\alpha$. Thus the conjecture is manifestly true for $J=1 / 2$.

\section{An Inequality for Entropy of Convolutions}

Lemmas 4 and 5 yielded a lower bound for $S^{\mathrm{cl}}$. Lemma 5 alone yields the following entropy inequality which, while not strictly related to coherent states, may be useful for related problems.

We first remark that if $f$ is a nonnegative function on $\mathbb{R}^{N}$ with $\int f(x) d x=1$, and if $f \in L^{s}\left(\mathbb{R}^{N}\right)$ for some $s>1$, then $\tilde{S}(f)$ is well defined in the sense that $\int_{f(x)>1} f(x) \ln f(x) d x<\infty . \tilde{S}(f)$ may be $+\infty$, however.

Theorem 6. Suppose $f$ and $g$ are nonnegative functions on $\mathbb{R}^{N}$ with $\int f=\int g=1$ and $f, g \in L^{s}\left(\mathbb{R}^{N}\right)$ for some $s>1$. Then $f * g$ has the same properties and

$$
\exp [2 \tilde{S}(f * g) / N] \geqq \exp [2 \tilde{S}(f) / N]+\exp [2 \tilde{S}(g) / N]
$$

(4.1) is equivalent to the following:

$$
\begin{aligned}
2 \tilde{S}(f * g) \geqq & 2 \lambda \tilde{S}(f)+2(1-\lambda) \tilde{S}(g) \\
& -N \lambda \ln \lambda-N(1-\lambda) \ln (1-\lambda)
\end{aligned}
$$

for all $\hat{\lambda} \in[0,1]$.

Corollary. $\tilde{S}(f * g) \geqq \frac{1}{2}[\tilde{S}(f)+\tilde{S}(g)+N \ln 2]$.

Remark. (4.1) is an equality if $f$ and $g$ are any two Gaussians of the form $f(x)$ $\sim \exp [-(x, M x)+(b, x)], g(x) \sim \exp [-\alpha(x, M x)+(c, x)]$ with $\alpha>0, b, c \in \mathbb{R}^{N}$ and $M$ positive definite.

Proof. By Lemma $5,(f * g) \in L^{p}\left(\mathbb{R}^{N}\right)$ for $p=1$ and for $p=s(2-s)^{-1}$. Hence $\tilde{S}(f * g)$ is well defined.

$(4.2) \Rightarrow(4.1)$ : Choose

$$
\begin{aligned}
\lambda= & \{\exp [2 \tilde{S}(f) / N]+\exp [2 \tilde{S}(g) / N]\}^{-1} \\
& \cdot \exp [2 \tilde{S}(f) / N] .
\end{aligned}
$$

$(4.1) \Rightarrow(4.2)$ : Geometric-arithmetic mean inequality. We now prove (4.2). In Lemma 5 , choose $p^{\prime}=r^{\prime} / \lambda, q^{\prime}=r^{\prime} /(1-\lambda)$, so that $1+r^{-1}=p^{-1}+q^{-1}$. By convexity, $f \in L^{s} \cap L^{1}$ implies $f \in L^{t}$ for $1 \leqq t \leqq s$ and $t \mapsto\|f\|_{t}$ is continuous for $t \in[0, s]$. For $r$ close enough $1, p, q<s$, so $f * g \in L^{r}$ and (2.5) holds. Furthermore, (2.5) is an equality for $r=p=q=1$ so one can take the right derivative at $r=1$. Without loss we can 
assume $\tilde{S}(f)$ and $\tilde{S}(g)<\infty$, for otherwise $\tilde{S}(f * g)=\infty$ by concavity and there is nothing to prove. For the same reason, one can assume $\tilde{S}(f * g)<\infty$. Next, we claim that if $F \in L^{1} \cap L^{s}, s>1$, and $\tilde{S}(F)<\propto$ then $\lim _{\varepsilon \downarrow 0} \varepsilon^{-1} \int F\left(1-F^{\varepsilon}\right)=\tilde{S}(F)$. To see this, let $A=\{x \mid F(x)<1\}$. Then for $x \in A, 0 \leqq 1-F(x)^{\varepsilon} \leqq-\varepsilon \ln F(x)$. For $x \in A^{c}$ and

$$
1 \leqq \varepsilon \leqq s-1, \quad 0 \leqq F(x)^{\varepsilon}-1 \leqq \varepsilon(s-1)^{-1}\left\{F(x)^{s-1}-1\right\} .
$$

The claim follows by dominated con'vergence. Thus, the right side of $(2.5)$ is differentiable at $r=1$ and Theorem 6 follows by explicit calculation. This calculation can be avoided noting that as $r$ varies, $p^{\prime} / q^{\prime}=$ const $=(1-\lambda) / \lambda$. As noted in Lemma 5, (if $N=1$, and hence for all $N$ ) (2.5) is saturated for the Gaussians $f(x)=\exp \left(-x^{2} / \lambda\right), g(x)=\exp \left(-x^{2} /(1-\lambda)\right)$, independent of $r$. But these Gaussians also give equality in (4.1).

\section{References}

1. Wehrl, A.: On the relation between classical and quantum-mechanical entropy. Rept. Math. Phys.

2. Schrödinger, E.: Naturwissenschaften 14, 664-666 (1926)

3. Bargmann, V.: Commun. Pure Appl. Math. 14, 187 -214 (1961): 20, 1-101 (1967)

4. Klauder,J. R.: Ann. Phys. (N.Y.) 11, 123 (1960)

5. Glauber, R.J.: Phys. Rev. 131, 2766 (1963)

6. Lieb,E.H. : Bull. Am. Math. Soc. 81, 1-13 (1975)

7. Beckner, W.: Ann. Math. 102, $159-182$ (1975)

8. Brascamp, H.J., Lieb,E.H.: Advan. Math. 20, 151-173 (1976)

9. Radcliffe,J. M. : J. Phys. A4, 313--323 (1971)

10. Kutzner, J.: Phys. Lett. A41, 475-476 (1972)

11. Atkins,P.W., Dobson,J.C.: Proc. Roy. Soc. (London) A321, $321-340$ (1971)

12. Arrechi,F.T, Courtens, E., Gilmore, R., Thomas, H.: Phys. Rev. A6, $2211-2237$ (1972)

13. Lieb, E.H.: Commun. math. Phys. 31, 327-340 (1973)

Communicated by J. Glimm

Received May 12, 1978 
